\title{
Apuntes metodológicos para el entrecruzamiento de fuentes históricas a través de sistemas de información geográfica: el caso de Santiago de Chile $(1650-1700)^{1}$
}

\author{
Claudio Ogass Bilbao², Rodrigo Torres Cañete 3 y Pablo Camus Gayán ${ }^{4}$
}

\begin{abstract}
RESUMEN
A través de la detallada explicación procedimental de un caso de estudio concreto de historia colonial urbana en el área de la Parroquia del Sagrario, correspondiente al casco histórico de la capital chilena, se exploran las posibilidades que ofrecen los Sistemas de Información Geográfica (SIG) al fichaje historiográfico de fuentes primarias. Este tipo de herramientas no solo permiten graficar productos finales. También son capaces de agregar nuevo valor a la información generada durante todo el proceso investigativo, por medio del entrecruzamiento de fuentes y el aprovechamiento analítico de los cambios que sufren los patrones de distribución, al instante en que se ingresan nuevos datos asociados a una referencia espacial determinada.
\end{abstract}

Palabras clave: SIG Histórico, Geografía Histórica, Santiago de Chile.

\begin{abstract}
Through procedural detailed explanation of a specific case study of urban colonial history in the area of the parish of El Sagrario, corresponding to the historical center of the Chilean capital, we explore the potential of Geographic Information Systems (GIS) in performing of historiographical tabs about primary sources. These tools not only allow graph the final products. They are also able to add new value to the information generated during the research process, through the crosslinking of sources and analytical use of the changes suffered by distribution patterns, at the same time which enter new data associated with a given spatial reference.
\end{abstract}

Key words: Historical GIS, Historical Geography, Santiago de Chile.

\footnotetext{
1 Proyecto "Evolución de la propiedad predial en el Centro Histórico de Santiago de Chile (Parroquia del Sagrario, 1700-1750)", financiado por el Fondo de Fomento y Apoyo a la Investigación de la Vicerrectoría de Investigación y Desarrollo de la Universidad Pedro de Valdivia. Agradecemos a Ana Bravo Jara, estudiante de la Universidad ARCIS, y Marjorie Araos Olea, estudiante de la Universidad Alberto Hurtado, por la ayuda brindada en la recopilación, catalogación y transcripción paleográfica de fuentes. Artículo recibido el 31 de mayo de 2012, aceptado
}

el 7 septiembre de 2012 y corregido el 30 de septiembre de 2012.

2 Estudiante de Magíster, Universidad de Chile (Chile). E-mail: cm.ogass.bilbao@gmail.com

3 Estudiante de Doctorado, Pontificia Universidad Católica de Chile (Chile).

E-mail: rjtorres@uc.cl

4 Instituto de Historia, Pontificia Universidad Católica de Chile (Chile).

E-mail: pcamusg@uc.cl 
El concepto Historical Geographic Information Systems (HGIS) designó, en un comienzo, a una infraestructura de datos espaciales (IDE) que contiene zonas que se modifican a lo largo de un período de tiempo significativo, y que se almacenan de tal forma que un usuario pueda formular, respecto de ellas, consultas cruzadas sobre lugares y fechas (Gregory \& Southall, 1998).

Una plataforma de HGIS se sustenta en el desarrollo de tecnologías creadas para procesar cierto tipo específico de información, compuesta por localizaciones determinadas sobre la superficie terrestre, que se vinculan a datos como nombres, funciones, población, elevación y temperatura, entre muchos otros. Este conjunto de técnicas integradas, conocidas como Sistemas de Información Geográfica (SIG), consideran también las capacidades profesionales necesarias para levantar y vaciar este tipo de contenidos, y permiten, en entornos digitales, realizar múltiples operaciones tales como crear, adquirir, almacenar, editar, transformar, analizar, visualizar e intercambiar esta información (Goodchild, 2000: 159). Los software de SIG, desde su creación en los años sesenta, han sido usados mayormente en las ciencias ambientales aplicadas, en disciplinas con intereses territoriales y como un apoyo a la gestión de sistemas de toma de decisiones (Morel-EdniBrown, 2009). Se basan en un modelo conceptualmente simple, que proporciona una ubicación en el espacio para cada contenido de la base de datos que se le vincula, y su origen se halla en la integración de los sistemas de gestión de estos registros tabulares con técnicas de cartografía informática (Gregory, 2005). Actualmente forman una industria multimiIlonaria de alcance mundial, que ha elevado considerablemente la calidad y accesibilidad de sus prestaciones (Morel-EdniBrown, 2009).

Debe reconocerse en el proyecto Great Britain Historical GIS, impulsado por la Universidad Queen Mary de Londres entre 1994 y 1999, a una iniciativa fundacional en el ámbito de los HGIS. Surgió con el objetivo de crear un gran atlas electrónico basado, mayormente, en información censal (Southall \& White, 1997). Desde 2000, esta plataforma es coordinada por la Universidad de Portsmouth. En la última década se han ejecutado proyectos afines tales como NHGIS, para Es- tados Unidos, que abarca el lapso 1790-2010 (Fitch \& Ruggles, 2003); HGIS Germany, que da cuenta de la evolución administrativa, demográfica y económica alemana para la época de su conformación nacional (Kunz, 2007); Belgium HGIS, que pone en valor registros municipales demográficos y económicos producidos entre 1800 y 1961 (Vanhaute, 2005), y CHGIS, un esfuerzo multinacional que reúne información serial de las unidades administrativas de China, desde la formación del primer Imperio, en 222 AC, hasta la proclamación de la República en 1911 (Bol, 2007).

De modo paralelo a estos desarrollos, una gran cantidad de publicaciones monográficas que no propenden, necesariamente, al desarrollo de una infraestructura de datos espaciales, se han inscrito en el concepto "HGIS". Este ha adquirido, durante la última década, identidad como metodología al interior de la investigación histórica (Knowles, 2002).

Quienes han llevado adelante estos estudios, alentados por una visión interdisciplinaria, suelen acompañar sus trabajos de una breve reseña sobre la historia y la lógica de funcionamiento de los SIG. Estas nociones elementales, si bien resultan evidentes para los ya iniciados en estas tecnologías, son todavía necesarias si se considera que dichas publicaciones están dirigidas, sobre todo, a un público muy enterado de cuestiones historiográficas, pero neófito en el ámbito de los SIG. Detrás de esta práctica recurrente subyace la esperanza de que tanto los historiadores como otros investigadores provenientes de las humanidades incorporen estas tecnologías de modo creciente al lenguaje y a la praxis de su disciplina.

Hasta el momento, los historiadores han usado escasamente estas herramientas, y su labor en el contexto de los estudios de HGIS ha servido, más bien, como mero insumo a las operaciones que realizan los expertos en geomática. Hay, al menos, tres razones para ello (Boonstra et al., 2004). La primera es una visión disciplinar restringida respecto de los mapas, usados como meros medios para la presentación de datos y no como herramientas analíticas. En segundo lugar, las lógicas internas de un software SIG así como su forma de importar datos resultan "muy exóticas" 
frente a las formas habituales de recopilar información historiográfica. Por último, estos programas no han logrado manejar de forma adecuada los cambios temporales ocurridos en el espacio geográfico.

Los aspectos teóricos subyacentes a las aplicaciones de SIG serán mejor aplicados con un sólido conocimiento temático (Gregory et al., 2003). Su uso trasciende el de un mero instrumento tecnológico, pues constituyen herramientas teóricas para el pensamiento (Owens, 2007) y que permiten actuar espacialmente (Buzai, 2007). En este contexto, es necesario que los historiadores conduzcan y diseminen sus propios estudios basados en SIG, para que establezcan, de acuerdo con su disciplina, un conjunto de "buenas prácticas" que no comprometan los métodos probados de investigación histórica (Gregory et al., 2003).

El presente trabajo acusa recibo de lo anterior, y propone el uso de un software de
SIG libre como herramienta para el fichaje historiográfico de un gran acervo documental vinculado territorialmente con el casco histórico de Santiago de Chile entre 1650 y 1700, específicamente, con la zona correspondiente a la jurisdicción de la Parroquia del Sagrario (Figura $\mathrm{N}^{\circ} 1$ ). Este período tiene un carácter refundacional, pues durante este lapso la ciudad consolidó su capitalidad, transformándose paulatinamente en la más prestigiosa, más poblada y más destacada del Reino de Chile (De Ramón, 2007).

Una motivación inicial para esta propuesta metodológica se encuentra en la obra "Evolución de la propiedad rural en el valle del Puangue" (Borde y Góngora, 1956). Esta investigación sobre la ocupación y geografía de suelos rurales, pionera en Chile, quiso estimular la "integración interna de la formación histórica con la geográfica" (Borde y Góngora, 1956: 17), a través de un estudio que combinó una enorme base documental con un acabado trabajo de terreno. Ello re-

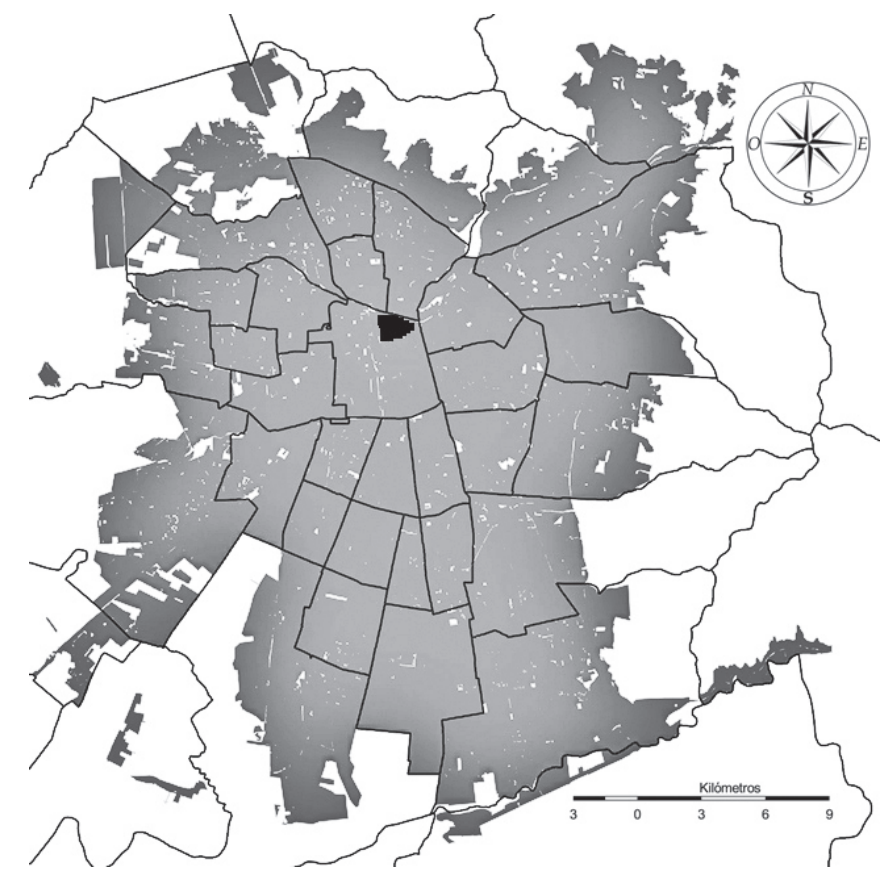

Fuente: Elaboración propia. 
cuerda las significativas palabras de Henri Berr al prologar "La Tierra y la Evolución Humana", de Lucien Febvre: "El problema de la influencia del medio no podría presentarse a un geógrafo puro. El geógrafo puro, el 'geógrafo geográfico' o no se preocupa de la historia o bien se dispone a incluirla en la geografía. Para tratar este problema complejo se necesita un geógrafo historiador, o, mejor, todavía, un historiador geógrafo y más o menos sociólogo por añadidura" (Berr, 1925: VI).

Lamentablemente, en Chile se han desarrollado pocos estudios vinculados a la ocupación del suelo y su distribución predial, y los trabajos realizados en torno a las relaciones entre la historia y la geografía han carecido de regularidad y coordinación entre sí. Constituyen felices excepciones algunos trabajos realizados para la zona conocida como "Norte Chico" (Galdames, 1964; Urbina, 1985) y la investigación intitulada "La Serena, un estudio de geografía urbana histórica" (Núñez y Labra, 1983), que se aproxima a la metodología planteada por Borde y Góngora.
Para el caso de Santiago de Chile, contamos con la monumental enumeración de los propietarios que tuvo cada uno de los solares de la ciudad durante el siglo XVI (Thayer Ojeda, 1905), un esfuerzo que fue recogido y emulado siete décadas después por el difunto historiador Armando de Ramón, quien, tras revisar 139 volúmenes del fondo documental de Escribanos de Santiago, entre 1675 y 1700, extrajo 1.481 escrituras de ventas de casas, que le permitieron reconstruir buena parte de la distribución predial de las parroquias del Sagrario, Santa Ana y San Isidro (De Ramón, 1974b; 1975).

\section{Valor renovado para fuentes conocidas}

Técnicamente, los mapas urbanos son los mapas históricos más sencillos de georrectificar, es decir, de alinear con las actuales coordenadas geográficas de un territorio, debido a que cubren un área relativamente pequeña (Knowles, 2005). Este proceso sería

Figura $N^{\circ} 2$

Parroquia del Sagrario, entre 1650 y 1700

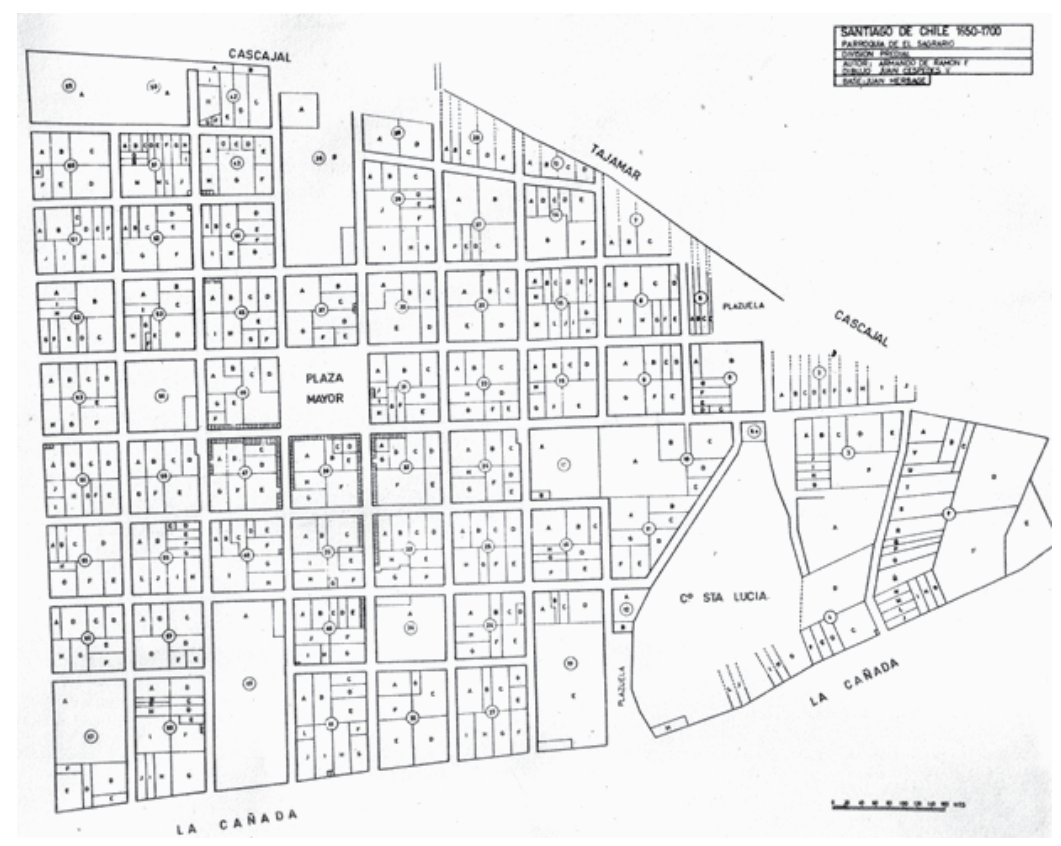

Fuente: De Ramón, 1974b. 
aún más sencillo si se aplicase en la mayoría de los cascos históricos de las ciudades latinoamericanas, cuyas trazas han experimentado escasas transformaciones a los largo del tiempo. Se añade a lo anterior que el proceso de deformación elástica (rubbersheeting), es decir, la corrección geométrica que permite la fusión de mapas de diferentes fuentes, recientemente ha progresado mucho gracias a los programas de SIG (Shimizu \& Fuse, 2003).

La base de la propuesta metodológica que aquí se presenta se halla en el plano que Armando de Ramón realizó para la parroquia El Sagrario entre 1650 y 1700 (Figura No 2), para representar la división predial estimada durante dicho período (De Ramón, 1974b). Para comenzar su procesamiento en un SIG, el primer paso fue georreferenciar este plano manzana a manzana, sobre la base de la traza urbana actual de Santiago. Este procedimiento garantiza apego a la fuente cartográfica empleada, pues, al usarse muy pocos puntos de control -usualmente las cuatro esquinas de una manzana común- se produce un buen ajuste a las áreas y formas de los diferentes predios, sin necesidad de recurrir a procesos técnicos más complejos, como el rubbersheeting por tramos basado en redes irregulares de triángulos (Triangular Irregular Network, TIN), o una transformación afín plana (Shimizu \& Fuse, 2003).

El trabajo de reconstrucción espacial hecho por Armando de Ramón ofrece una seria dificultad, pues comprime, en una sola representación, los cambios ocurridos durante medio siglo. Es un mosaico de yuxtaposiciones de distintos momentos. Sin embargo, gracias a los SIG, es posible introducir la variable temporal

Figura $\mathrm{N}^{\circ} 3$

Tópicos obtenidos a partir del padrón de Armando de Ramón

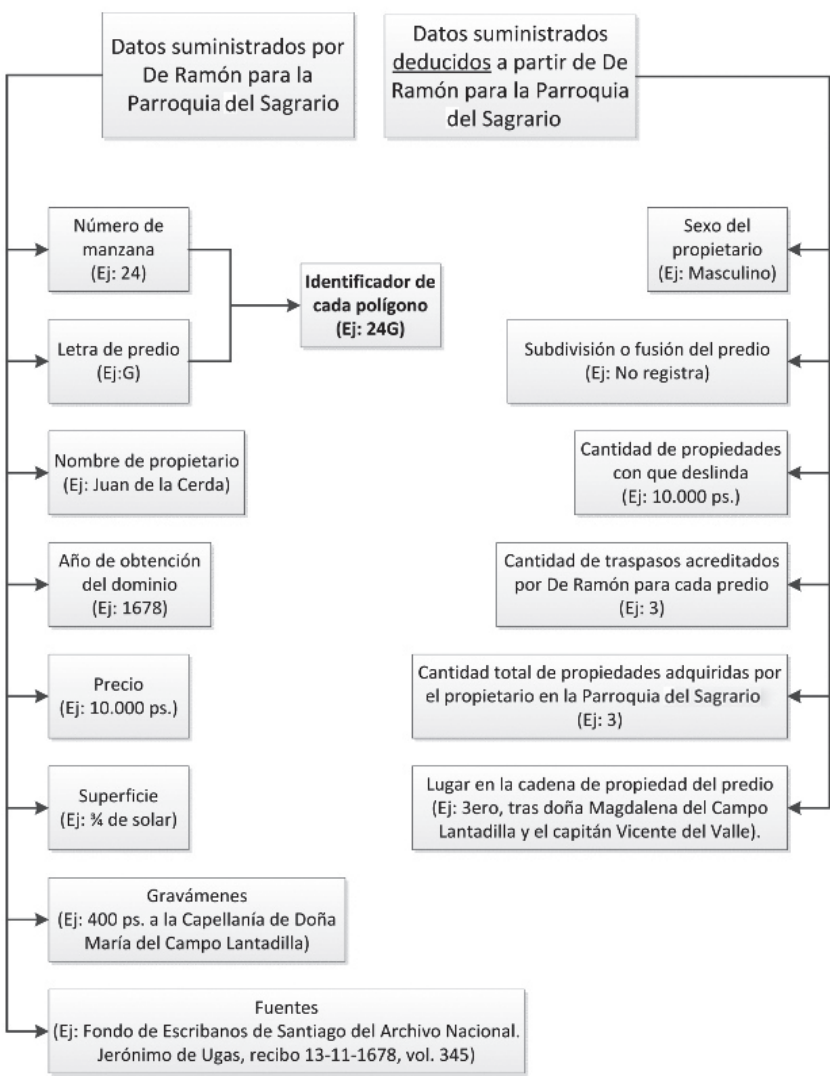

Fuente: Elaboración propia. 
a este dibujo, que intentó sintetizar y congelar en una misma imagen, numerosas modificaciones ocurridas tanto en la superficie de los predios como en su ocupación y propiedad.

Detrás de cada polígono dibujado por De Ramón subyace lo que él llamó una "relación pormenorizada" de los bienes raíces representados, que consiste en un desglose ordenado por cuadras en que da cuenta de ciertos atributos de cada predio: su historia, que corresponde a la secuencia más o menos completa de los traspasos escriturados de dominio; sus deslindes, en que se mencionan los propietarios de las tierras colindantes; su superficie, habitualmente medida en solares; su precio, que incluye montos y modalidades de pago; los gravámenes, estipulando monto y entidad favorecida, y las fuentes documentales desde las cuales se extrajeron -o se dedujeron- los datos. Para sistematizar estos contenidos a través de un $\mathrm{SIG}^{5}$, es necesario consolidar la información suministrada predio a predio, en un único registro tabular. Su número de filas será igual a la cantidad de predios representados -755 en el caso del Sagrario- y su número de columnas expresará los distintos tópicos inventariados (Figura $N^{\circ}$ $3)$, según sea el interés del investigador.

Una de las cualidades más evidentes de los Sistemas de Información Geográfica es que son capaces de generar nueva información a partir de operaciones realizadas con los datos ya existentes. Aplicada esta característica al trabajo de De Ramón se pueden obtener diversas cartografías que iluminan su estudio y que le otorgan mayor poder explicativo, pues a través de la fuerza integradora de los SIG, recursos muy diferentes entre sí pueden unirse para crear contextos amplios y ricos para el descubrimiento histórico y para el análisis espacial (Gregory et al., 2003). A modo de ejemplo, se generaron dos mapas temáticos sencillos para las condiciones

\footnotetext{
5 Pocos proyectos del tipo HGIS usan software libre, pero los resultados de diversas iniciativas internacionales demuestran la factibilidad de su uso (Rubio Campillo, 2007), por lo que, para la presente experiencia, se ocupó el Sistema de Información Geográfica de la Generalitat Valenciana, gvSIG. Para los usuarios latinos, este programa resulta fácil de aprender por el idioma, por su interfaz intuitiva y un muy bien planeado sistema de menús emergentes.
}

técnicas actuales ${ }^{6}$. El primero muestra la distribución de los predios pertenecientes a los mayores propietarios del Sagrario, es decir, quienes poseyeron cinco o más inmuebles durante el período estudiado (Figura $\mathrm{N}^{\circ}$ 4). El segundo da cuenta de la cantidad de traspasos acreditados por De Ramón para cada predio y permite ilustrar el dinamismo del mercado de tierras de la época (Figura $N^{\circ} 5$ ). En él se aprecia un patrón en que las zonas periféricas, sobre todo al norte y al este, manifiestan menos intercambios que el segmento suroeste. Para hacer ambas representaciones, solo fue necesario someter los datos tabulados a la función de hojas de cálculo "CONTAR.SI", que cuenta las celdas de un rango que tienen datos y que cumplen con determinado criterio. Esto se hizo para los nombres de los propietarios, en el primer caso, y para los identificadores de cada polígono, en el segundo.

Se debe tener en cuenta que el objetivo del trabajo efectuado por De Ramón no fue conseguir la descripción física de la ciudad de Santiago, aunque esta fue un medio para comprender la estratificación social urbana de la capital (Góngora, 1975), que logró representar cartográficamente a través de una zonificación de seis sectores en el plano correspondiente al año 1700 (De Ramón, 1978). Este historiador, conocedor de sus documentos, advirtió que trabajando a esta escala de análisis espacial, más compatible con barrios que con propiedades individuales, podría obtener conclusiones significativas de la enorme cantidad de fuentes primarias que logró acumular. La aplicación de los SIG a este conjunto de datos podría devolver nuestra atención a la escala predial.

\section{Los documentos de una vida: el caso de Juan de la Cerda}

Así como la propuesta metodológica de Armando de Ramón fue sistematizada con la

\footnotetext{
6 Es pertinente recordar que cuando Tomás Thayer Ojeda y Armando de Ramón hicieron sus monumentales trabajos, no contaban sino con medios analógicos para vaciar y ordenar su información, y que como muchos historiadores, perdieron, ignoraron, o sobrecargaron aspectos de los componentes espaciales de sus respectivas investigaciones (Gregory et al., 2003).
} 
expectativa de que fuese replicada en otras trazas urbanas coloniales (De Ramón, 1978), este apartado propone una ruta de exploración documental que puede servir como modelo a futuras investigaciones. Se trata de la búsqueda de los rastros históricos de Juan de la Cerda y Contreras, oidor de la Real Audiencia que en los años 1676, 1704 y 1709 ejerció como alcalde ordinario del Cabildo, y que en 1710 fue elegido auditor general del ejército. Nació en Santiago en 1638, del matrimonio del maestre de campo don Alonso de la Cerda y la señora Teresa Méndez y Contreras. Se graduó en Lima de licenciado en cánones y leyes, recibiendo el título de abogado. Fundó un mayorazgo en una escritura de 9 de octubre de 1703. Murió el 12 de septiembre de 1713 (Medina, 1906; Figueroa, 1897). A través de un estudio demográfico regional (Mellafe y Salinas, 1988) se sabe que era propietario de una de las cuatro grandes haciendas que existían en La Ligua a comienzos del siglo XVIII, y que lo propio sucedía con su esposa, Mariana de Hermúa, dueña de la hacienda El Ingenio, cuya historia de traspasos comienza en 1552, cuando fue dada a Gonzalo de los Ríos por Pedro de Valdivia. La tenencia de ambos territorios se gestó en mercedes del siglo XVI.

"La estancia del Ingenio -que incluía la estancia de San Lorenzo- se mantuvo en poder de la familia De los Ríos hasta 1685, en que fue subastada públicamente, por la causa ejecutiva que siguieron los acreedores de Catalina de los Ríos, por Juan de Hermúa, quien pagó por ella \$ 15.038:2 1/2. Este la traspasó en 1695 a Juan de la Cerda, abogado de la Real Audiencia, quien la vinculó en mayorazgo en 1703, avaluándose en \$12.000 los terrenos que la componían" (Mellafe y Salinas, 1988: 97).

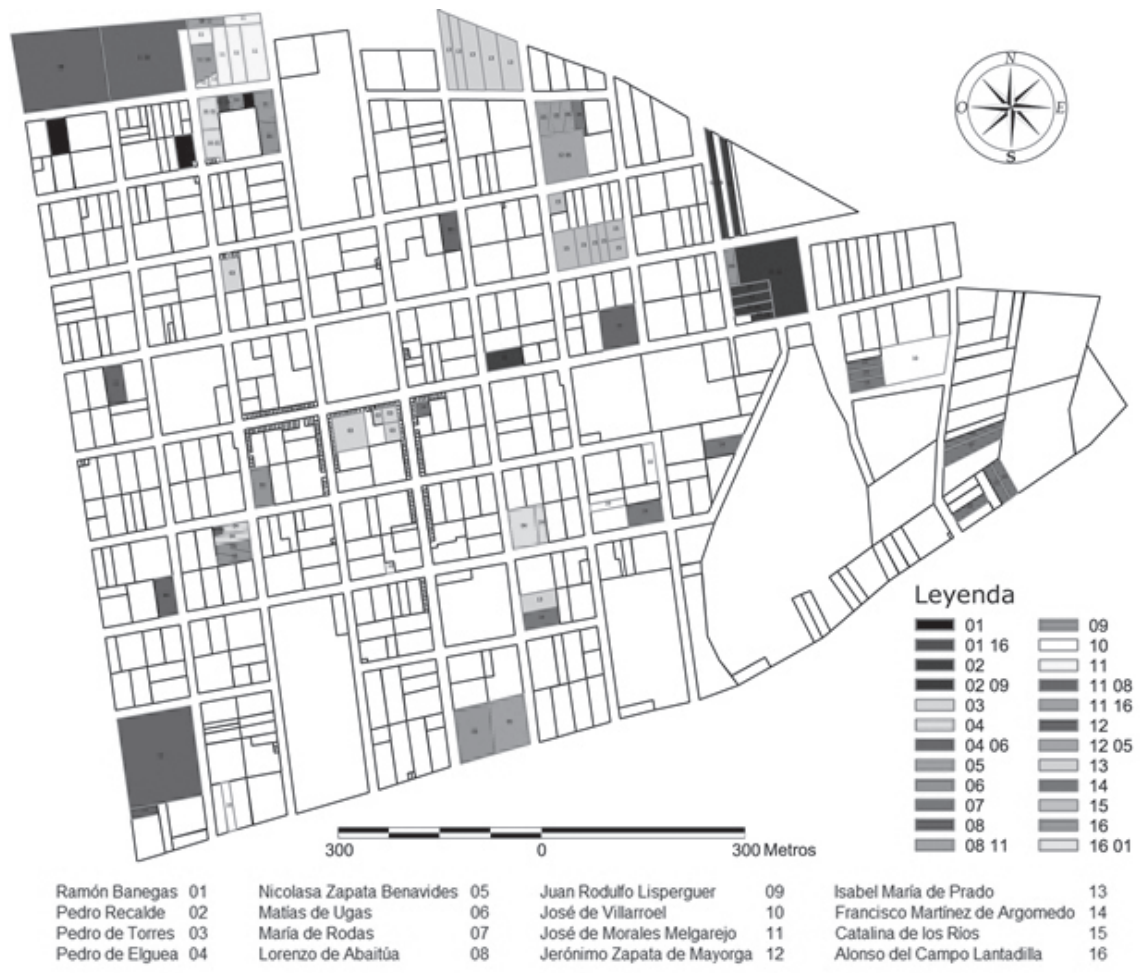

Fuente: Elaboración propia. 
Figura $\mathrm{N}^{\circ} 5$

Cantidad de traspasos acreditados por De Ramón para cada predio entre 1650 y 1700

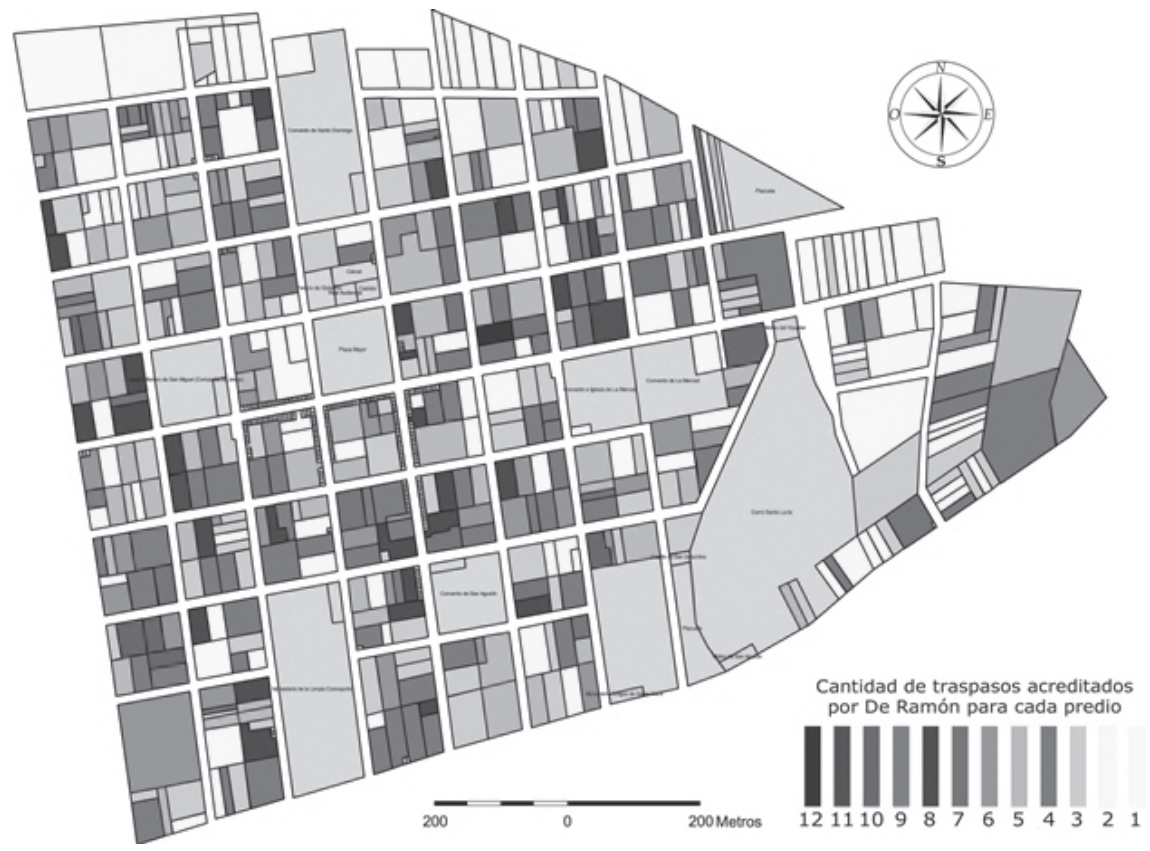

Fuente: Elaboración propia.

Figura $\mathrm{N}^{\circ} 6$

Propiedades de Juan de la Cerda y su círculo social cercano en la Parroquia del Sagrario, entre 1650 y 1700

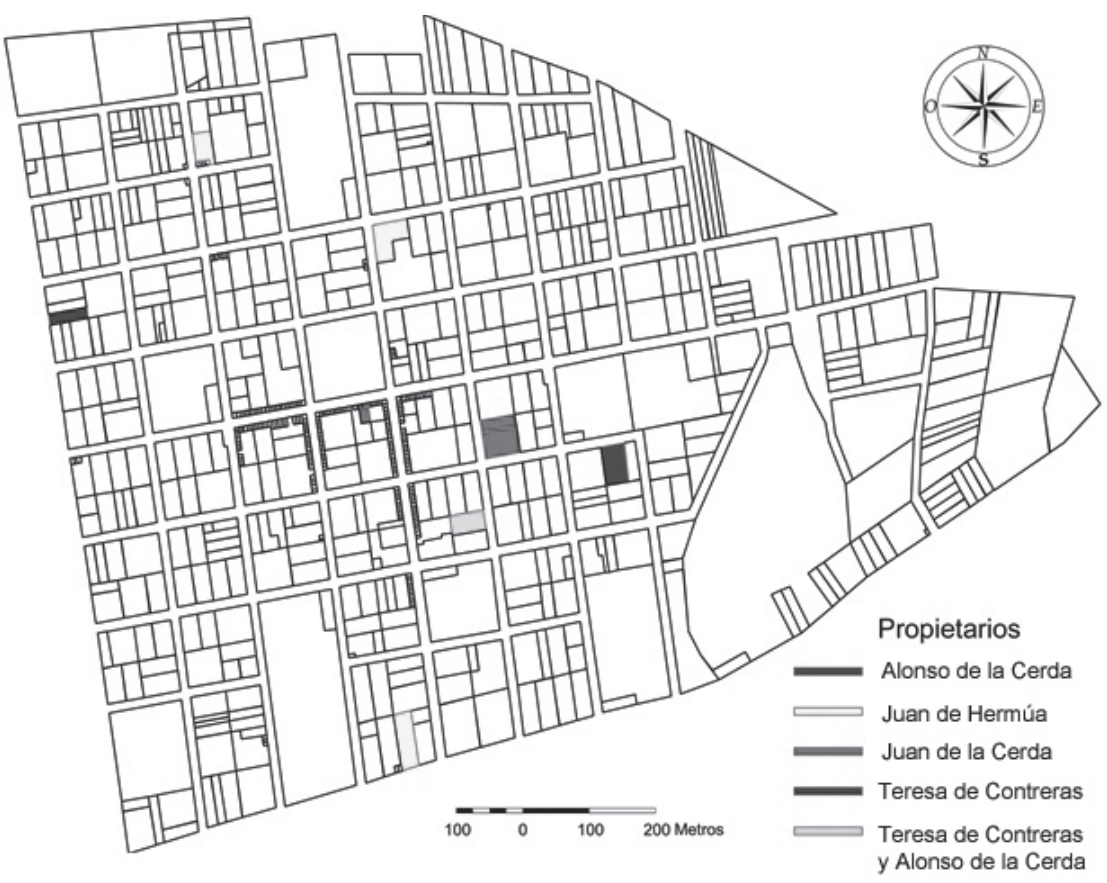

Fuente: Elaboración propia. 
Además de estos rastros bibliográficos, absolutamente preliminares, el nombre de Juan de la Cerda figura en los registros de De Ramón para la Parroquia del Sagrario, por lo que se pueden visualizar tanto sus propiedades como las de los integrantes de su círculo social que han sido mencionados hasta acá (Figura $\mathrm{N}^{\circ}$ 6). Este es el punto de partida del entrecruzamiento de fuentes, que no se acaba en el mero conocimiento de los predios que estuvieron bajo el dominio de estas personas, pues cada nuevo antecedente y nombre mencionado permitirá retratar tanto a nuestro protagonista como a parte de su red social y de sus vínculos espaciales. Ello requiere, más que saber de SIG, conocer cómo explorar los fondos documentales, y qué información se puede extraer de ellos.

Comencemos por los fondos disponibles en el Archivo del Arzobispado de Santiago (AAS) (Figura $N^{\circ} 7$ ). Durante el siglo XIX, algunos curas intervinieron los libros de bautismo, matrimonio y defunción de sus predecesores para organizarlos y hacer más operativo el uso de estos documentos en el futuro. Desconocemos la causa de este ejercicio que, sin embargo, es agradecido por la historia. Esto es posible de inferir por las diferencias de grafías y de color de las tintas existentes entre los registros coloniales y los decimonónicos, que se superponen a la manera de un palimpsesto.

Entre las tareas que facilitaron las consultas se halla la realización de índices onomásticos. Es altamente probable que esta empresa se haya debido a Rafael Molina, cura y vicario de la Parroquia del Sagrario en 1868 (Cf. Prieto del Río, 1922: 481-482). Algunos libros de bautismos -por ejemplo, los números 12 y 13 de Castas- contienen anotaciones tales como: "Este libro tiene ciento sesenta i siete fojas útiles inclusas las mutiladas. Santiago, julio 6 de 1868. Rafael Molina". La letra del prelado coincide con la de los mencionados índices, lo que refrenda la inferencia aquí expresada.

Figura $\mathrm{N}^{\circ} 7$

Documentos y tipos de información del fondo parroquial del archivo del Arzobispado de Santiago

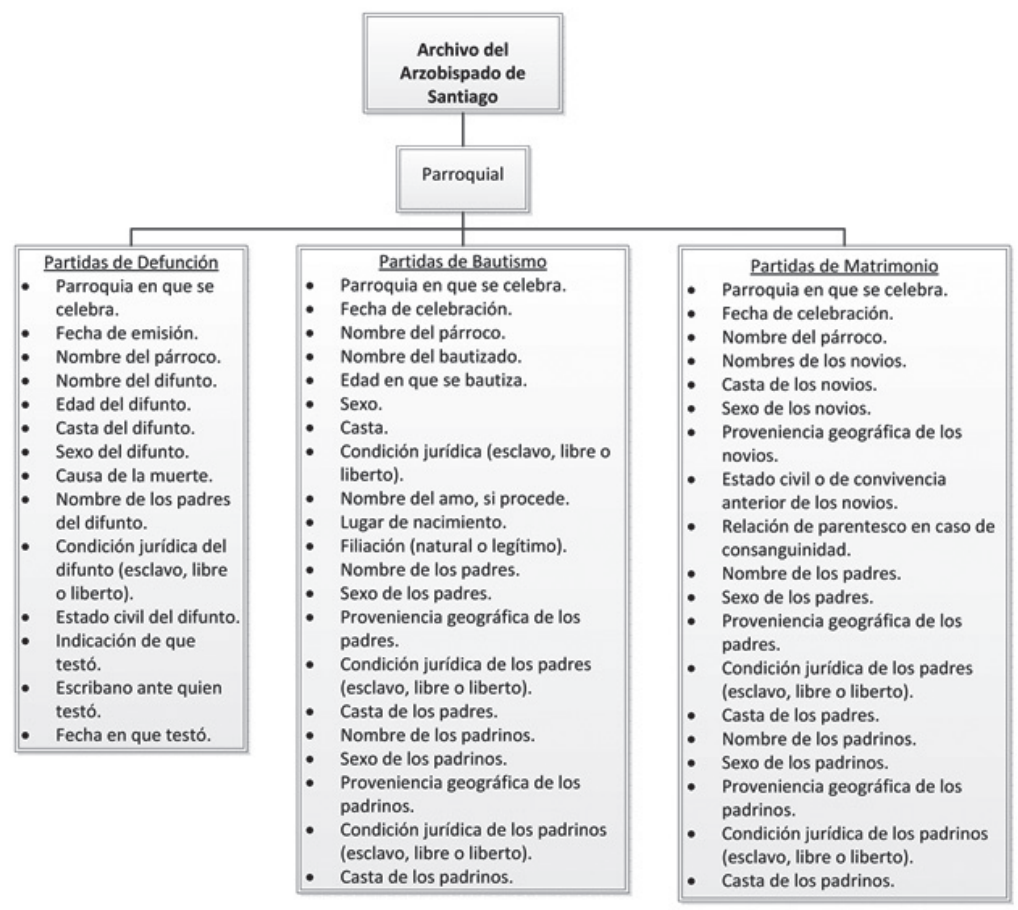

Fuente: Elaboración propia. 
Sea cual fuere el caso, esta feliz ocurrencia permite que la ubicación de documentos parroquiales sea extremadamente expedita para los siglos XVI, XVII y XVIII. Solo se debe buscar un apellido -siguiendo el criterio de transmisión utilizado en el siglo XIX- para conocer tanto la familia de un determinado personaje, como de sus esclavos. Ante cualquier duda y omisión de los curas, lo que es frecuente, es posible cotejar la información con otro tipo de fuentes, principalmente, con las partidas de matrimonios. Así se establecen conexiones seguras y consolidadas de los vínculos familiares investigados.

En el fondo Parroquial del AAS se buscó el bautismo de Juan de la Cerda en el libro $N^{\circ} 5$ de Españoles, que correspondería a las coordenadas temporales de su nacimiento según sus biógrafos. Los genealogistas saben que, en general, esta información se puede obtener con facilidad. Lamentablemente, este libro solo contiene fragmentos y, lo más probable, es que se haya perdido. La búsqueda partió mal, pero sí están disponibles la partida de Matrimonio de Juan de la Cerda Contreras con Mariana de Hermúa Contreras ${ }^{7}$, la partida de Velación de Juan de la Cerda con Mariana de Hermúa ${ }^{8}$ y la defunción de Mariana de Hermúa ${ }^{9}$. Curiosamente, figuran dos defunciones correspondientes al mismo licenciado Juan de la Cerda ${ }^{10}$, que deberán ser escrutadas con atención. Mejor suerte se corre con los bautizos de Juan Pedro Francisco Javier, Antonio Apolinardo, Nicolás Apolinardo, Tomás Nicolás, Carlos José, Francisco y José de la Cerda Hermúa, hijos de este matrimonio ${ }^{11}$.

7 Santiago, 18 de febrero de 1672, Archivo del Arzobispado de Santiago (En adelante AAS), Parroquia del Sagrario, Libro de Matrimonios № 1, f. 65.

8 Santiago, 12 de febrero de 1673, AAS, Parroquia del Sagrario, Libro de Matrimonios № 1, f. 65-81.

9 Santiago, 22 de noviembre de 1725. Archivo del Arzobispado de Santiago, Libro de Defunciones № 1 , Parroquia del Sagrario, f. 131.

10 Santiago, 14 de septiembre de 1713. AAS, Libro N ${ }^{\circ}$ 1 Defunciones, Parroquia del Sagrario, f. 37 vta.-69 vta, y Santiago, 22 de agosto de 1717. AAS, Libro № 1 Defunciones, Parroquia del Sagrario, 69 vta.

11 Santiago, 30 de octubre de 1672. AAS, Parroquia del Sagrario, Libro de Bautismos (de Españoles) No 9, f. 59; Santiago, 15 de agosto 1674. AAS, Parroquia del Sagrario, Libro de Bautismos (de Españoles) № 9, f. 93 vta.; Santiago, 15 de agosto 1674. AAS, Parro-
También se encuentran los bautizos de ciertos indios aucas y encomendados de la familia Cerda Hermúa: Sebastián Cerda, indio auca; María de las Cabezas Cerda, auca infiel; Francisca de la Cerda y Juana Lorenza de la Cerda, indias libres del servicio de Juan de la Cerda; José de la Cerda, indio de la encomienda de Juan de la Cerda; Rafael Cerda, indio; Josefa Cerda, india del servicio de Juan de la Cerda; Juan del Cristo y Manuela Cerda ${ }^{12}$. Finalmente, se encuentran gran cantidad de bautizos relativos a los esclavos negros y mulatos de esta familia, correspondientes a Lázara Francisca Ignacia de la Cerda, María Josefa Cerda, María Mercedes de la Cerda y N. de la Cerda, mulatas; Ignacio de la Cerda, Juan Martín de la Cerda, Pablo Josef de la Cerda, Santiago de la Cerda y Francisco Javier de la Cerda, mulatos; Juana de los Santos Cerda y Marcela Ignacia, negras; Juan Josef de la Cerda y Pedro Juan Cerda, negros, e Isabel de la Cerda, Juan Francisco de las Llagas, Juana de Dios de la Cerda, María de Hermúa y Pascuala de la Santísima Trinidad ${ }^{13}$.

quia del Sagrario, Libro de Bautismos (de Españoles) № 9, f. 93 vta.; Santiago, 20 de septiembre de 1675. AAS, Parroquia El Sagrario, Libro de Bautismos (de Españoles) No 9, f. 115 vta.; Santiago, 15 de noviembre de 1676. AAS, Parroquia del Sagrario, Libro de Bautismos (de Españoles) № 9, f. 136; Santiago, 7 de enero de 1678. AAS, Parroquia del Sagrario, Libro de Bautismos (de Españoles) No 9, f. 162 vta., y Santiago, 23 de agosto de 1681. AAS, Parroquia del Sagrario, Libro de Bautismos (de Españoles) $\mathrm{N}^{\circ}$ 9, f. 209.

12 Santiago, 12 de enero de 1674. AAS, Parroquia del Sagrario, Libro de Bautismos $N^{\circ} 10$ (de Castas), f. 26 vta.; Santiago, 5 de septiembre de 1677 . AAS, Parroquia del Sagrario, Libro de Bautismos $N^{\circ} 10$ (de Castas), f. 103 vta.; Santiago, 10 de septiembre de 1677. AAS, Parroquia del Sagrario, Libro de Bautismos No 10 (de Castas), f. 103 vta.; Santiago, 28 de agosto de 1679. AAS, Parroquia del Sagrario, Libro de Bautismos N 10 (de Castas), f. 131.; Santiago, 8 de julio de 1689. AAS, Parroquia del Sagrario, Libro de Bautismos № 10 (de Castas), f. 212 vta.; Santiago, 10 de enero de 1692. AAS, Parroquia del Sagrario, Libro de Bautismos $N^{\circ} 10$ (de Castas), f. 235; Santiago 29 de junio de 1702. AAS, Parroquia del Sagrario, Libro de Bautismos No 12 (de Castas), f. 129; Santiago, 2 de septiembre de 1702. AAS, Parroquia del Sagrario, Libro de Bautismos No 12 (de Castas), f. 133 vta, y Santiago, 28 de enero de 1707. AAS, Parroquia del Sagrario, Libro de Bautismos $\mathrm{N}^{\circ}$ 13 (de Castas), f. 35 vta.

${ }^{13}$ Santiago, 31 de agosto de 1708. AAS, Parroquia del Sagrario, Libro de Bautismos No 13 (de Castas), f. 59 vta.; Santiago, 20 de abril de 1709. AAS, Parroquia 
Provistos de este acervo de nombres con sus respectivos atributos, suministrados por el formato propio de los documentos bautismales, el trabajo proseguirá en los fondos del Archivo Nacional Histórico (ANHCH) (Figura $N^{\circ} 8$ ), específicamente, en el de Escribanos de Santiago.

Para continuar exitosamente con nuestro rastreo onomástico, contamos con dos potentes auxiliares que agilizan la búsqueda. Entre 1913 y 1930, el historiador Tomás Thayer Ojeda publicó sus tres tomos de la Guía para facilitar la consulta del Archivo de Escribanos, en la cual realiza una enumeración de las dotes, testamentos e inclusive de los inventarios de bienes de los vecinos que concurrieron a escriturar durante el período colonial. En tanto, el Instituto Chileno de Investigaciones Genealógicas ha generado un índice digital de este fondo, que contiene la ubicación de más de 1.500 protocolos.

del Sagrario, Libro de Bautismos $\mathrm{N}^{0} 13$ (de Castas), f. 100 vta.; Santiago, 18 de septiembre de 1700 . AAS, Parroquia del Sagrario, Libro de Bautismos $\mathrm{N}^{\circ}$ 12 (de Castas), f. 78.; Santiago, 25 de diciembre de 1678. AAS, Parroquia del Sagrario, Libro de Bautismos № 10 (de Castas), f. 124.; Santiago, 16 de abril de 1709. AAS, Parroquia del Sagrario, Libro de Bautismos No 13 (de Castas), f. 69 vta.; Santiago 23 de junio de 1701. AAS, Parroquia del Sagrario, Libro de Bautismos № 12 (de Castas), f. 101 vta.; Santiago, 18 de febrero de 1686. AAS, Parroquia del Sagrario, Libro de Bautismos No 10 (de Castas), f. 191 vta. Santiago, 23 de julio de 1706. AAS, Parroquia del Sagrario, Libro de Bautismos № 13 (de Castas), f. 28 vta.; Santiago, 31 de enero de 1702. AAS, Parroquia del Sagrario, Libro de Bautismos $N^{\circ} 12$ (de Castas), f. 119 vta.; Santiago, 11 de noviembre de 1710 . AAS, Parroquia del Sagrario, Libro de Bautismos $N^{\circ}$ 13 (de Castas), f. 95 vta.; Santiago, 27 de enero de 1709. AAS, Parroquia del Sagrario, Libro de Bautismos № 13 (de Castas), f. 65 vta.; Santiago, 30 de octubre de 1711. AAS, Parroquia del Sagrario, Libro de Bautismos $N^{\circ} 13$ (de Castas), f. 104 vta.; Santiago, 11 de julio de 1700. AAS, Parroquia del Sagrario, Libro de Bautismos No 12 (de Castas), f. 72 vta.; Santiago 15 de julio de 1702. AAS, Parroquia del Sagrario, Libro de Bautismos № 12 (de Castas), f. 130; Santiago, 4 de octubre de 1700 . AAS, Parroquia del Sagrario, Libro de Bautismos № 12 (de Castas), f. 79 vta.; Santiago, 16 de marzo de 1707. AAS, Parroquia del Sagrario, Libro de Bautismos $N^{\circ} 13$ (de Castas), f. 39 vta.; Santiago, 20 de mayo de 1703. AAS, Parroquia del Sagrario, Libro de Bautismos $\mathrm{N}^{\circ} 12$ (de Castas), f. 153 vta., y Santiago, 3 de junio de 1698. AAS, Parroquia del Sagrario, Libro de Bautismos $\mathrm{N}^{\circ}$ 12 (de Castas), f. 17 vta.
En esta colección nos volvemos a encontrar con una sorpresa. Al igual que con los bautizos, figuran dos testamentos a nombre del licenciado Juan de la $\mathrm{Cerda}^{14}$, siendo el segundo en una fecha muy posterior, a la que se supone corresponde a su defunción. Ello obliga a verificar consistencias entre los documentos y a revisar la posibilidad de un alcance de nombres. Además se hallan dos testamentos de Mariana de Hermúa ${ }^{15}$, el Inventario de la Estancia "El Ingenio"16, la dote de Mariana de Hermúa ${ }^{17}$, y los codicilos de ésta y de Juan de la Cerda ${ }^{18}$.

La mayor variedad documental se expresa en los fondos de Capitanía General y Real Audiencia, pues corresponden a la realización de juicios de distinta índole. En el primero se halla un litigio sobre concesión de nuevo título de merced de ciertas tierras en la provincia de Concepción ${ }^{19}$, un pleito de Juan Andrés de Ustáriz con Juan de la Cerda a raíz de una causa pasada entre este y Juan Francisco González ${ }^{20}$, una disputa sobre un título de tierras en Itata ${ }^{21}$, un litigio con José de la Cruz sobre la confirmación de una encomienda de indios 22 , un juicio por el pago de media anata ${ }^{23}$, un pleito sobre la venta del

${ }^{14}$ Santiago, 4 de diciembre de 1705. Archivo Nacional Histórico (En adelante $\mathrm{ANHCH}$ ), Fondo de Escribanos de Santiago, volumen 582, fs. 279 vta., y Santiago, 19 de agosto de 1723. ANHCH, Fondo de Escribanos de Santiago, volumen 523, f. 161 vta.165.

15 Santiago, 19 de agosto de 1723. ANHCH, Fondo de Escribanos de Santiago, volumen 523, f. 161 vta., y Santiago, 19 de agosto de 1723. ANHCH, Fondo de Escribanos de Santiago, volumen 617, f. 402 vta.

16 Inventario de los bienes de la estancia El Ingenio, La Ligua. Santiago, 9 de octubre de 1703. ANHCH, volumen 440, fs. 118-131.

17 Santiago, 23 de diciembre de 1672. ANHCH, Fondo de Escribanos de Santiago, volumen 335, fs. 489491 vta.

18 Santiago, 3 de diciembre de 1714. ANHCH, Fondo de Escribanos de Santiago, volumen 583, f. 343., y Santiago, 14 de diciembre de 1711. ANHCH, Fondo de Escribanos de Santiago, volumen 582, fs. 298.

19 Santiago, 15.10.1683-17.10.1683, AHNCH, Capitanía General, volumen 32, pieza $15^{a}$, fs. 323-327.

20 Santiago, 20.3.1711. ANHCH, Capitanía General, volumen 204, pieza $98^{a}$, fs. 273-275 vta.

21 Santiago, 1683. AHNCH, Capitanía General, volumen 478, pieza $40^{\mathrm{a}}$, fs. 79 vta.-82.

22 Santiago, s/f. AHNCH, Capitanía General, volumen 521, pieza $47^{\circ}$, f. 268.

23 Santiago, 22.4.1706. AHNCH, Capitanía General, volumen 580 , pieza $236^{\mathrm{a}}$, f. 307. 


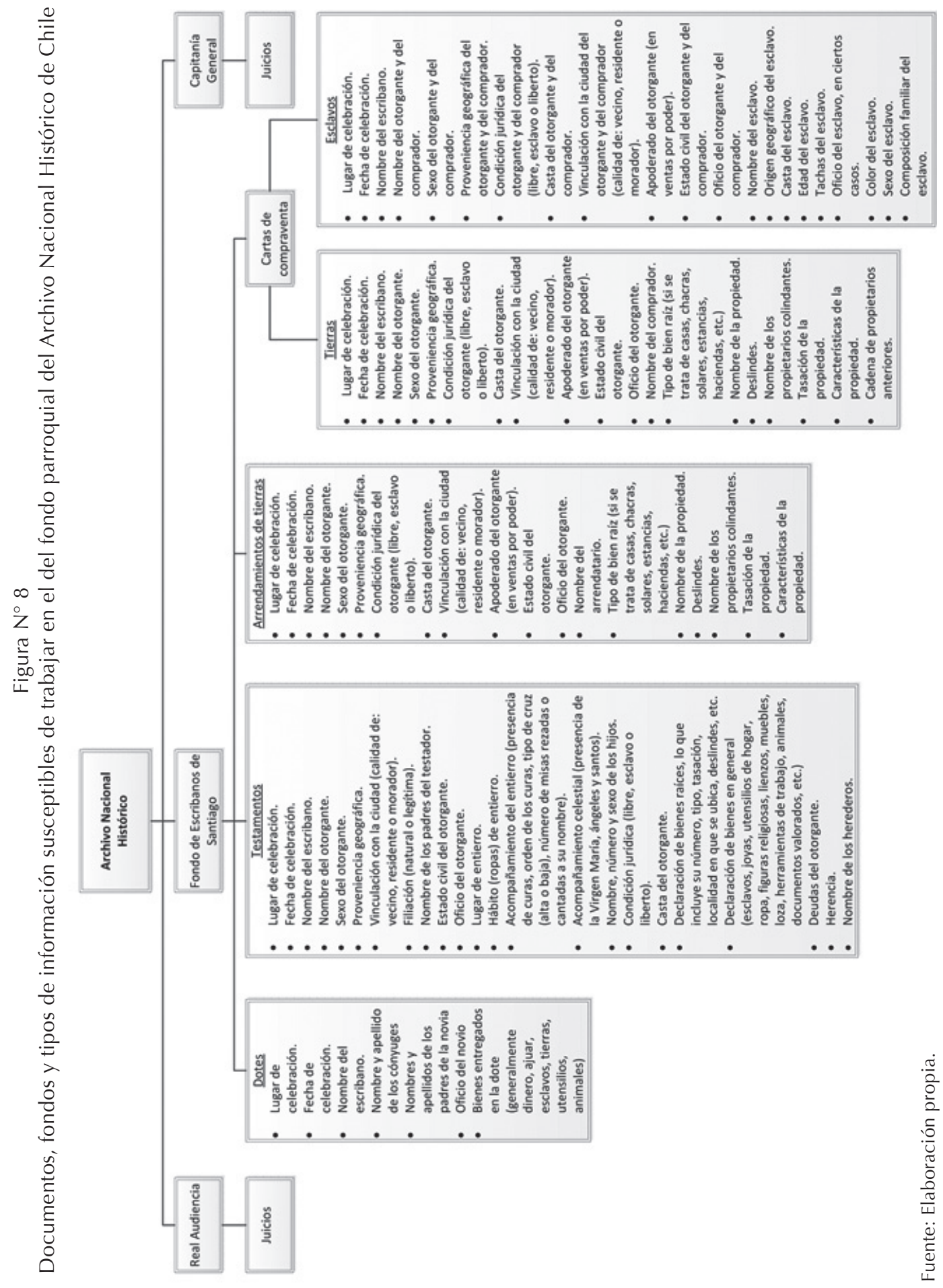


oficio de receptor de cámaras ${ }^{24}$, un escrito relativo a una Encomienda ${ }^{25}$, y una causa en que José Cisternas solicita merced de una mina en Copiapó ${ }^{26}$.

Aparecen también cuatro documentos fundamentales para esclarecer posibles conflictos de identidad, pues están fechados años después de la fecha de muerte atribuida al sujeto de nuestra búsqueda, y que además lo vinculan a un cargo del que no se tenía noticia. Se trata de una presentación de Gabriel Cano de Aponte sobre si Juan de la Cerda continúa en su cargo de corregidor ${ }^{27}$, un expediente sobre una querella entre Alonso Astudillo y el corregidor Juan de la Cerda por el abasto de nieve de esta ciudad ${ }^{28}$, un pleito con Inés de Olivera por despojo de una acequia ${ }^{29}$, y una presentación sobre algunos puntos relativos al gobierno ${ }^{30}$. Finalmente, se encuentra una causa de Marcos de la Cerda con su padre, Luis de la Cerda, en que solicitan para sí la encomienda de indios que poseyó Juan de la Cerda, su abuelo y padre, respectivamente ${ }^{31}$. En el fondo de Real Audiencia se encuentran, para este caso, documentos de composición más compleja. En primer lugar, hay un expediente formado sobre la oposición que hacen al cargo de Protector general de Indios los oidores don Juan de la Cerda y don Juan del Corral Calvo de la Torre, y el abogado de la Real Hacienda don Francisco Ruiz, en 1707, y que contiene, además, una Real Cédula de 16 de septiembre de 1673 que trata de la forma en que debe proveerse dicho empleo ${ }^{32}$. También se halla

\footnotetext{
24 Santiago, 26.10.1684-22.11.1697. AHNCH, Capitanía General, volumen 616 , pieza $11^{\text {a }}$, fs. $136-177$ vta.

25 Santiago, 6.9.1681, AHNCH, Capitanía General, volumen 475 , pieza ${ }^{a}$, fs. $18-20$.

26 Santiago, 31.10.1711. AHNCH, Capitanía General, volumen 189, pieza 102, fs. 220-222 vta.

27 Santiago, 28.11.1724. AHNCH, Capitanía General, volumen 1037, pieza $76^{\text {a }}$, fs. 221-221 vta.

${ }^{28}$ Santiago, 26.9.1725. AHNCH, Capitanía General, volumen 1000, pieza $3^{\text {a }}$ fs. 39-47 vta.

${ }^{29}$ Santiago, 31.12.1724. AHNCH, Capitanía General, volumen 176, pieza $15^{\text {a }}$, fs. 80-160 vta.

30 Aculeo, 10.2.1724. AHNCH, Capitanía General, volumen 677, pieza 15, f. 105-120.

31 San Bartolomé de Chillán, 1.11.1695, AHNCH, Capitanía General, volumen 523, pieza 20a , fs. 172 177.

$32 \mathrm{AHNCH}$, Fondo de la Real Audiencia de Santiago, volumen 482 , pieza $6^{\text {a }}$.
}

un juicio de partición de los bienes quedados por la muerte de Tomás de la Cerda y de doña Beatriz de Águila, su mujer, que incluye sus respectivos testamentos, más el de doña Feliciana Riberos y una serie de inventarios y tasaciones de los bienes allí expresados ${ }^{33}$. Lamentablemente, faltan algunas hojas al final de este legajo. Finalmente, este fondo nos ofrece un juicio por cobro de pesos con Juan Carretero de la Vega ${ }^{34}$, unos autos con Juan Roco y Campofrío sobre derechos de agua ${ }^{35}$, de 1704, y una presentación de 1699 del Procurador General de los Indios por la defensa de Melchor y Juan, indios de la encomienda de Juan de la Cerda, "sobre que no les inquiete en su reducción" ${ }^{36}$.

Es manifiesto que las vertientes posibles de información vinculada a un solo nombre y a un solo predio son extensas. Cómo se acoten las bases de datos resultantes dependerá de los objetos de estudio que se escojan, y de las variables espaciales que sean investigadas.

\section{Proyección de los SIG en la investigación historiográfica}

Si los Sistemas de Información Geográfica han de ser una herramienta para la historia, deben dejar de aparecer solo al final de las investigaciones, donde suelen descollar con cartografías espléndidas. Es hora de integrarlos en el aparato epistemológico de los estudios sobre el pasado, en los procesos de pensamiento, entendiendo que los SIG no han producido solo una revolución tecnológica, sino que están ocasionando una revolución intelectual en que el espacio geográfico es central, pues se desarrolla en torno a los conceptos de localización, distribución, asociación, interacción y evolución espacial (Buzai, 2007).

Ya se ha destacado que los historiadores deben encontrar sus propias formas de uso

\footnotetext{
$33 \mathrm{AHNCH}$, Fondo de la Real Audiencia de Santiago, volumen 1035, pieza 1 ${ }^{\mathrm{a}}$, fs. 1-91.

$34 \mathrm{AHNCH}$, Fondo de la Real Audiencia de Santiago, volumen 1122, pieza $2^{\mathrm{a}}, 73$ fojas.

$35 \mathrm{AHNCH}$, Fondo de la Real Audiencia de Santiago, volumen 2118, pieza $1^{\mathrm{a}}$, fs. 1-8.

$36 \mathrm{AHNCH}$, Fondo de la Real Audiencia de Santiago, volumen 2771 , pieza $5^{\mathrm{a}}, 18$ fojas.
} 
de estas herramientas, que sean consecuentes con su tradición disciplinar, en lugar de trasplantar técnicas producidas en otras áreas del conocimiento. En este sentido, no deben preocupar tanto los problemas técnicos que se susciten por la integración de los SIG en la investigación histórica: lo esencial es discutir cómo formular y responder a las preguntas en un contexto de datos e instrumentos limitados (Gregory et al., 2003). Pensar lo contrario equivale a intercambiar la relación sujetoobjeto (Buzai, 2007), lo que conduce a una nueva fuente de alienación, en que la información contenida en ciertos objetos técnicos concretos induce a obedecer lógicas ajenas (Santos, 2000). El riesgo es relevante, si se considera que muchos investigadores están encantados con la oportunidad de traducir su investigación en imágenes, lo cual es difícil cuando las fuentes son, esencialmente, los documentos escritos. (Rebelatto e Freitas, 2012).

Las tecnologías que aquí se han tratado posibilitan nuevas formas de análisis, que suelen ser eficientes y capaces de encontrar correlaciones imposibles de hallar con otros métodos. Tampoco sustituyen a la narrativa, pero ayudan a su construcción. Además, permiten el cruce de un gran número de fuentes, facilitando tanto la observación de patrones como la inexistencia de los mismos (Rebelatto e Freitas, 2012). Por otra parte, como herramienta y técnica combinada, los SIG proporcionan herramientas para leer el paisaje (read the landscape) de forma no habitual y para obtener información de fuentes tradicionalmente ajenas a los estudios espaciales (Morel-EdniBrown, 2009).

\section{Referencias bibliográficas}

AGUILAR-ROBLEDO, M. Formation of the Miraflores Hacienda: Lands, Indians, and Livestock. In: Eastern New Spain at the End of the Sixteenth Century. Journal of Latin American Geography, 2003, Vol. 2, №1, p. 87-103.

AGUILAR-ROBLEDO, M. y TORRESMONTERO, M.G. Ambiente y cambio ambiental: ¿ejes para deconstruir y (re)construir a la historia ambiental? Vetas. Revista del Colegio de San Luis, 2005, Vol. VII, N¹9, p. 9-33.
ARNOLD, D. La naturaleza como problema histórico: el medio, la cultura y la expansión de Europa. México: FCE, 2001.

BERR, H. Prólogo. Acción del medio y explotación de la tierra. In: FEBVRE, L. La tierra y la evolución humana. Introducción geográfica a la Historia. México: Uteha, 1925.

BODENHAMER, D. J. History and GIS: implication for the discipline. In: KNOWLES, A.K. \& HILLIER, A. Placing history: how maps, spatial date, and GIS are changing historical scholarship. Redlands: ESRI Press, 2007.

BOL, P.K. The China Historical Geographic Information System (CHGIS). Choices Faced, Lessons Learned. In: Conference on Historical Maps and GIS. 23 y 24 de agosto de 2007.

BOONSTRA, O.; BREURE, L. \& DOORN P. Past, present and future of historical information science. Historical Social Research, 2004, Vol. 108, p. 4-114.

BORDE, J. y GÓNGORA, M. Evolución de la propiedad rural en el Valle de Puangue. Santiago de Chile: Editorial Universitaria, 1956.

BUZAI, G. D. Dilemas de la relación Geografía-SIG entre la disciplina, la interdisciplina y la transdisciplina. GeoFocus, 2007, No 7, p. 5-7.

DE RAMÓN, A. La ciudad de Santiago entre 1650 y 1700. Ensayo de Metodología para su estudio e interpretación. Documento de Trabajo $N^{\circ} 77$. Santiago de Chile: Centro de Desarrollo Urbano y Regional de la Universidad Católica de Chile, 1974a.

DE RAMÓN, A. Santiago de Chile, 16501700, Parte I. Historia, 1974b, №13, p. 93374.

DE RAMÓN, A. Santiago de Chile, 16501700, Parte II. Historia, $1975, N^{\circ} 14$, p. $97-$ 270.

DE RAMÓN, A. Historia urbana: una metodología aplicada. Buenos Aires: Clacso, 1978. 
DE RAMÓN, A. Santiago de Chile, 1541 - 1991. Historia de una sociedad urbana, Santiago de Chile: Catalonia, 2007.

FEBVRE, L. La tierra y la evolución humana. Barcelona: Editorial Cervantes, 1925.

FIGUEROA, P.P. Diccionario biográfico de Chile. Tomo I. Santiago de Chile: Imprenta y Encuadernación Barcelona, 1897.

FITCH, C. A. \& RUGGLES S. Building the National Historical Geographic Information System. Historical Methods, 2003, Vol. 36, $\mathrm{N}^{\circ} 1$, p. 41-51.

GALDAMES, J. La Serena y su evolución urbana. Revista Chilena de Historia y Geografía, 1964, N¹32, 134-195

GÓNGORA, M. Urban Stratification in Colonial Chile. The Hispanic American Historical Review, 1975, Vol. 55, N³, p. 421-448.

GOODCHILD, M.F. New horizons for the social sciences: geographical information systems. Canadian Journal of Policy Research, 2000, Vol. 1, N¹, p. 158-161.

GREGORY, I.N.; KEMP, K. y MOSTERN, R. Geographical Information and historical research: Current progress and future directions. History and Computing, 2003, N $N^{\circ} 13$, p. 7-21.

GREGORY, I.N. A place in history: A guide to using GIS in historical research. Belfast: Centre for Data Digitization and Analysis of Queens University, 2005.

GREGORY, I.N. \& ELL, P. S. Historical GIS: technologies, methodologies and scholarship. Cambridge: Cambridge University Press, 2008.

GREGORY, I. N. \& SOUTHALL, H. Putting the Past in Its Place: the Great Britain Historical GIS. In: CARVER, S. (editor). Innovations in GIS 5. Londres: Taylor y Francis, 1998, p. 210-211.

KNOWLES, A.K. Introducing Historical GIS. In: Knowles, A.K. (editor). Past Times, Past Place. GIS for history. Redlands: ESRI Press, 2002.
KNOWLES, A. K. Emerging Trends in Historical GIS. Historical Geography, 2005, Vol. 33, p. 7-13.

KNOWLES, A.K. Report on national historical GIS projects. Historical Geographer, 2005, Vol. 33, p. 134-58.

KUNZ, A. Fusing Time and Space: The Historical Information System HGIS Germany. International Journal of Humanities y Arts Computing, 2007, Vol. 1, № 2, p. 111-122.

MEDINA, J. T. Diccionario Biográfico CoIonial. Santiago de Chile: Imprenta Elzeviriana, 1906.

MELLAFE, R. y SALINAS MEZA, R. Sociedad y población rural en la formación de Chile actual. La Ligua, 1700-1850. Santiago de Chile: Ediciones Universidad de Chile, 1988.

MOREL-EDNIEBROWN, F. EarthTextSpaceTime: making historical sources in cities available through the agency of GIS. Computer Applications to Archaeology, 2009, p. 22-26.

NÚÑEZ, J. y LABRA, P. La Serena, un estudio de geografía urbana histórica. Revista Geográfica de Chile: Terra Australis, 1983, № 27, p. 134-195.

OWENS, J. B. Toward a GeographicallyIntegrated, Connected World History: Employing Geographic Information Systems (GIS). History Compass, 2007, Vol. 5, p. 2014-2040.

PRIETO DEL RÍO, L.F. Diccionario Biográfico del Clero Secular de Chile: 1535-1918. Santiago de Chile: Imprenta Chile, 1922.

REBELATTO, M. e FREITAS, F. Desafios e possibilidades ao uso de Sistemas de Informação Geográfica na história. En: $5^{\circ}$ Encontro Escravidão e Liberdade no Brasil Meridional, 11 a 13 de mayo de 2012, Porto Alegre, UFRGS.

RUBIO CAMPILLO, X. Las Herramientas SIG Libre en la investigación histórica. A I Jornadas de SIG Libre. Girona: Universitat, 2007. 
SANTOS, M. La naturaleza del espacio. Barcelona: Editorial Ariel, 2000.

SHIMIZU, E. \& FUSE, T. Rubber-sheeting of historical maps in GIS and its application to landscape visualization Old-Time Cities: Focusing on Tokyo of the Past. In: In Proceedings of the 8th International Conference on Computers in Urban Planning and Urban Management, 2003, Vol. 11A-3.

SOUTHALL, H.R. \& WHITE, B. Creating an Electronic Historical Atlas of Britain. Geocal, 1997, No 16, p. 3-6.

THAYER OJEDA, T. Santiago durante el siglo XVI. Constitución de la propiedad urbana y noticias biográficas de sus primeros pobladores. Santiago de Chile: Imprenta Cervantes, 1905.
URBINA, R. Los repartimientos de chacras en las poblaciones del Norte Chico de Chile durante el siglo XVIII. Cuadernos de Historia 6, 1985, p. 17-31.

VANHAUTE, E. The Belgium Historical GIS. Historical Geography, 2005, Vol. 33, p. 136-139.

WORSTER, D. Doing environmental history. 1988. In: WORSTER, D. (editor). The ends of the Earth. Perspectives on modern environmental history. Cambridge: Cambridge University Press, 1994.

ZUSMAN, P. Unidad y diversidad en la Geografía Histórica. XI Encuentro de Geógrafos de América Latina (EGAL). Bogotá, 26 al 30 de marzo de 2007. 